\title{
A novel variant in ALMSI in a patient with Alström syndrome and prenatal diagnosis for the fetus in the family: A case report and literature review
}

\author{
CONG ZHOU ${ }^{1,2}$, YUANYUAN XIAO ${ }^{1,2}$, HANBING XIE ${ }^{1,2}$, SHANLING LIU ${ }^{1,2^{*}}$ and JING WANG ${ }^{1,2^{*}}$ \\ ${ }^{1}$ Department of Obstetrics and Gynecology, West China Second University Hospital; ${ }^{2}$ Key Laboratory of Birth Defects and \\ Related Diseases of Women and Children, Ministry of Education, Sichuan University, Chengdu, Sichuan 610041, P.R. China
}

Received February 5, 2020; Accepted July 15, 2020

DOI: $10.3892 / \mathrm{mmr} .2020 .11398$

\begin{abstract}
Alström syndrome (AS) is a type of monogenic syndromic ciliopathy disease. The main clinical features of AS include cone-rod malnutrition, sensorineural hearing loss, metabolic dysfunctions and multiple organ failure, which are caused by mutations of Alström syndrome protein 1 (ALMS1) gene. The current study aimed to identify pathogenic variants in a Chinese patient with AS and to review the relevant literature. Genomic DNA extracted from a 10-year-old male with AS was evaluated using a disease-targeted gene panel. According to the bioinformatics analysis, the current study identified a novel frameshift mutation in exon 8 (c.2988_2989del, p.T996fs) and a rare nonsense mutation in exon 10 (c.9535C $>$ T, p.R3179*) of the ALMS1 gene. Both parents were heterozygous carriers of this gene. To the best of our knowledge, these mutations have not been reported in normal population databases. According to the criteria of the American College of Medical Genetics and Genomics, the mutations were pathogenic. Based on these findings, amniotic fluid sample was used for prenatal diagnosis of the couple's fetus, and it was observed that the fetus carried c.9535C $>$ T, and not c.2988del. During the follow-up duration of $>2$ years of the fetus, it was confirmed that he was a healthy male. The results of the present study identified two compound heterozygous ALMS1 mutations in a patient with the symptoms of Alström syndrome and reported a novel ALMS1 variant which expands the spectrum of ALMS1 variants in AS.
\end{abstract}

Correspondence to: Dr Shanling Liu or Dr Jing Wang, Department of Obstetrics and Gynecology, West China Second University Hospital, Sichuan University, 20 Renmin South Road, Chengdu, Sichuan 610041, P.R. China

E-mail: sunny630@126.com

E-mail: hhwj_123@163.com

*Contributed equally

Key words: Alström syndrome, Alström syndrome protein 1, mutation, disease-targeted gene panel, next generation sequencing, prenatal diagnosis

\section{Introduction}

Alström syndrome (AS; OMIM 203800) was first reported in 1959 (1). AS is a multisystem disease with cone-rod retinal dystrophy, which can lead to visual impairment, sensorineural hearing loss, obesity and hyperinsulinemia (2). Other disease phenotypes that may seriously affect prognosis and survival include endocrine disorders, dilated cardiomyopathy and restrictive pulmonary disease (1-3).

AS is caused by mutations in the Alström syndrome protein 1 (ALMS1) gene that is located on chromosome 2p13 (4). The total length of ALMS1 is $224 \mathrm{~kb}$, comprising 23 exons, which were identified in 2002 (5). The ALMS1 consists of 4,169 amino acids (NM_015120.2). ALMS1 has been reported to be widely expressed and localized to the centrosomes and basal bodies of ciliated cells of tissues including the central nervous, photoreceptor, cardiopulmonary, reproductive, endocrine and urological system (6-8). Although the exact biological function of ALMS1 remains obscure, current evidence suggests that the functions include maintaining ciliary function, intracellular transport and adipocyte differentiation (6-8). Early diagnosis of AS is usually based on the phenotype and by direct sequencing analysis of the ALMS1 gene (8), but is complicated by the progressive and non-simultaneous onset of the principal symptoms $(2,6,8)$.

In the present study, two compound heterozygous mutations were identified in the proband, which presented symptoms of low vision, hearing loss and childhood obesity. Based on these results, prenatal diagnosis for the fetus was provided for this family and at the same time, relevant literature to explore the main clinical characteristics, diagnosis and treatments for AS was reviewed.

\section{Patients and methods}

Patient. A Chinese woman who was 4 months pregnant, visited the Prenatal Diagnosis Center of West China Second University Hospital of Sichuan University (Chengdu, China) for genetic consultation in May 2018. The patient reported that her first child was a 10-year-old male, who was diagnosed with retinitis pigmentosa at another hospital. The proband was obese since he was 6 months old, his height was $140 \mathrm{~cm}$ and 


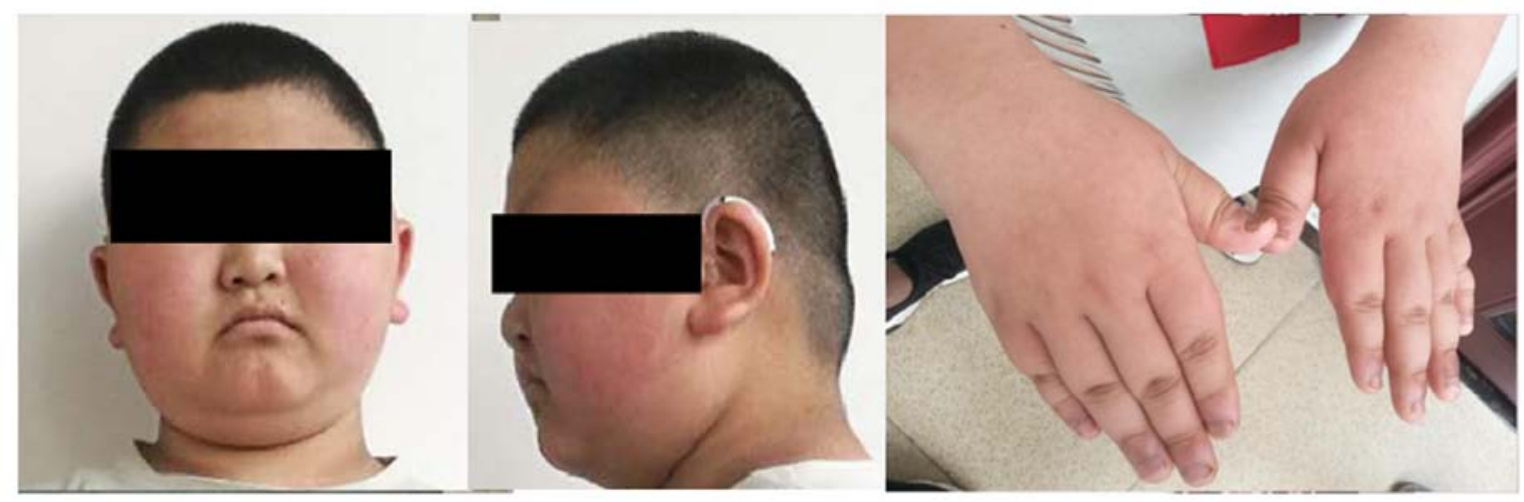

Figure 1. Clinical symptoms of the patient. The patient had narrow blepharoplasty, round face, sparse hair, wide and thick hand with short, thick fingers.

I
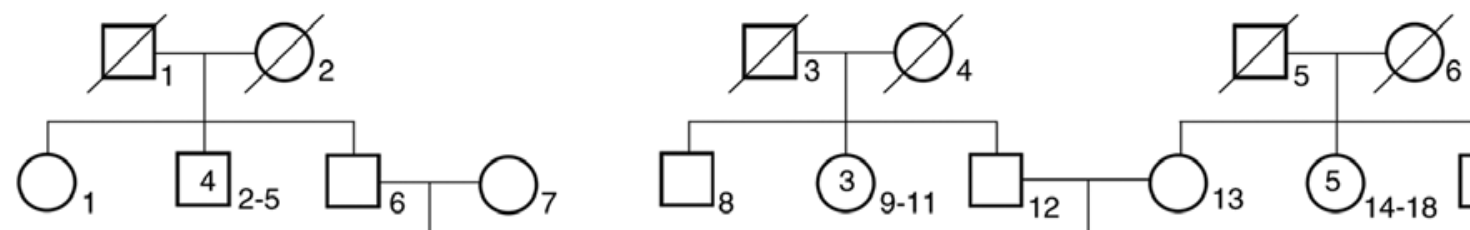

II

III

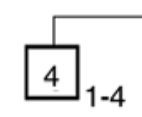

IV

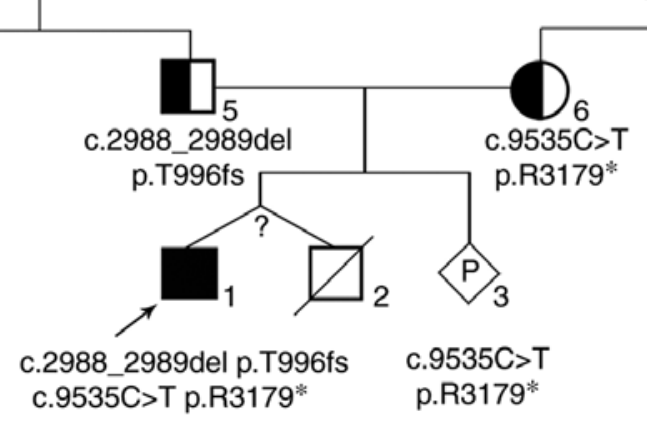

Figure 2. Pedigree of the patient's family. Arrow indicates the patient.

his weight was $65 \mathrm{~kg}$ (body mass index was $33 \mathrm{~kg} / \mathrm{m}^{2}$ ). He had unique facial features, including narrow blepharoplasty, round face and sparse hair (Fig. 1). Additionally, he had wide and thick feet with short, thick fingers and toes. He displayed photophobia and poor vision in the first year of life, which eventually led to partial blindness at the age of 7-8 years. Bilateral hearing loss occurred at the age of 7 years. According to the aforementioned clinical phenotype, the doctor's clinical diagnosis was suspected AS.

In addition, the proband had a twin brother. The twins were born prematurely by caesarean section, so they were born at the same time. However, the mother could not provide characteristics at birth, including their birth length, weight and head circumference. Unfortunately, the proband's twin brother died of fever and diarrhea 50 days after birth, leaving no specimen for further laboratory testing. The parents of the proband were healthy, unrelated adults and the mother denied any existence of family history of congenital malformation and genetic diseases, and also denied the exposure to teratogenic environment during pregnancy. The pedigree of the patient's family is described in Fig. 2. The current study was approved by the Medical Ethics Committee of West China Second University Hospital of Sichuan University (Chengdu, China) and written informed consent was obtained from the parents of the patient.
Genetic testing. The peripheral blood samples of the patient and his parents were collected and the genomic (g)DNA was extracted using a QIAamp DNA Blood Mini kit (Qiagen GmbH) according to the manufacturer's protocol. The quality and concentration of gDNA were assessed with the NanoDrop 1000 (Thermo Fisher Scientific, Inc.) and 1\% agarose gel electrophoresis. For the proband, a disease-targeted gene panel (selected genes sequenced by a next-generation sequencing method) was employed using the MGP011 Targeted Exome Capture kit (MyGenostics, Inc.) according to the manufacturer's instructions; the kit targets 171 genes known to cause metabolic diseases (including ALMSI gene). AmpliSeq ${ }^{\mathrm{TM}}$ Library PLUS (96 Reactions) for Illumina ${ }^{\circledR}$ reagent (cat. no. 20019102; Illumina, Inc.) was used for enrichment libraries. The purified and enriched DNA libraries were sequenced using the Illumina Nextseq 500/550 medium flux V2 kit (300 cycles; cat. no. FC-404-2004; Illumina, Inc.) and NextSeq 500 platform (Illumina, Inc.) to generate paired-end reads for 150 cycles per read. The loading concentration of DNA libraries was $1.8 \mathrm{pM}$, which was measured using qPCR analysis (7500 Fast Dx Real-Time PCR Instrument, Thermo Fisher Scientific, Inc.) according to the manufacturer's protocol. DNA libraries quantification was performed by amplifying the set of six pre-diluted DNA Standards and diluted library samples by qPCR, using the KAPA SYBR FAST qPCR 


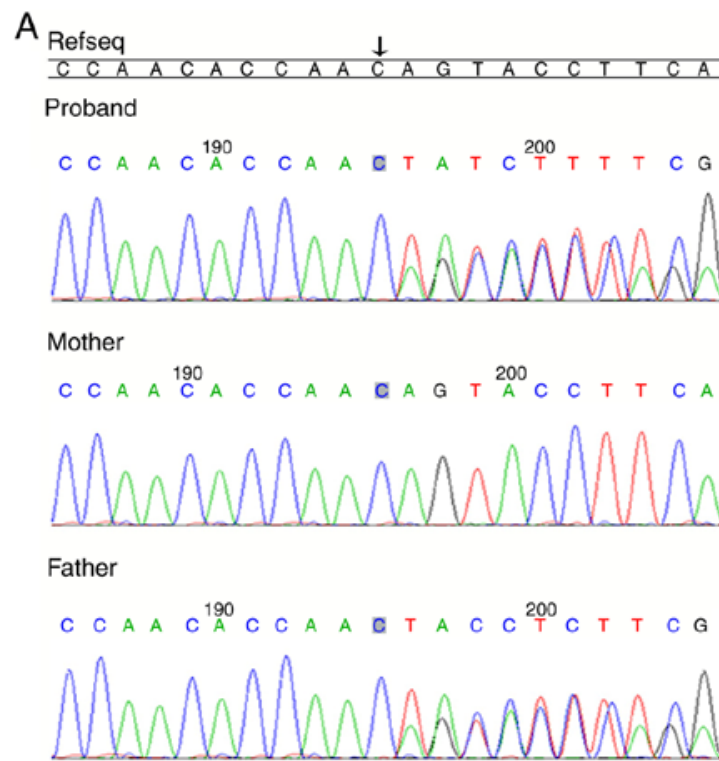

Fetus (amniotic fluid cells)

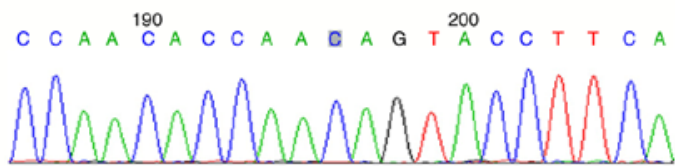

B

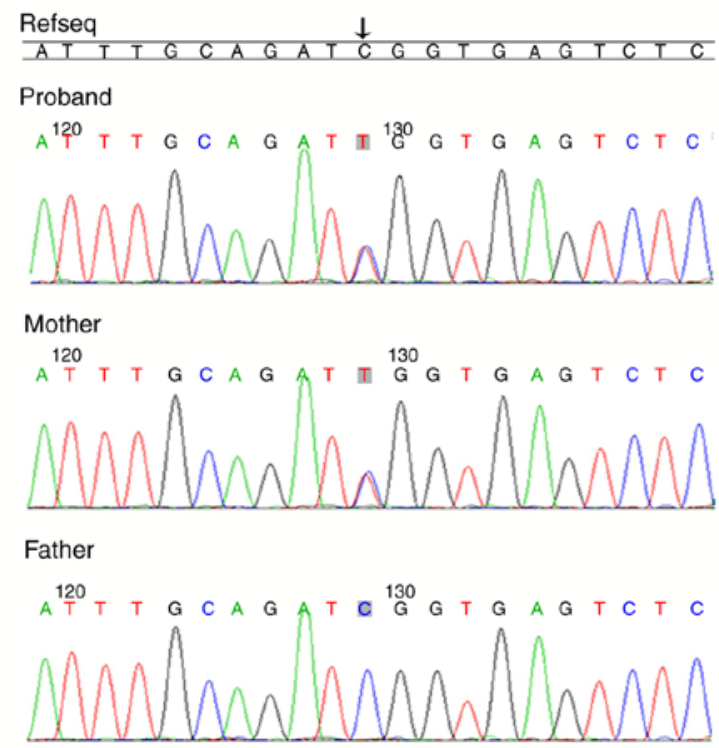

Fetus (amniotic fluid cells)

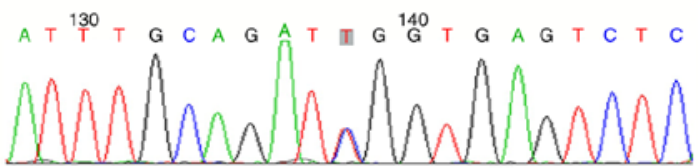

Figure 3. Confirmation of two mutations in the family members by Sanger sequencing. (A) c.2988_2989delAG (p.T996fs) mutation. (B) c.9535C >T (p.R3179*) mutation.

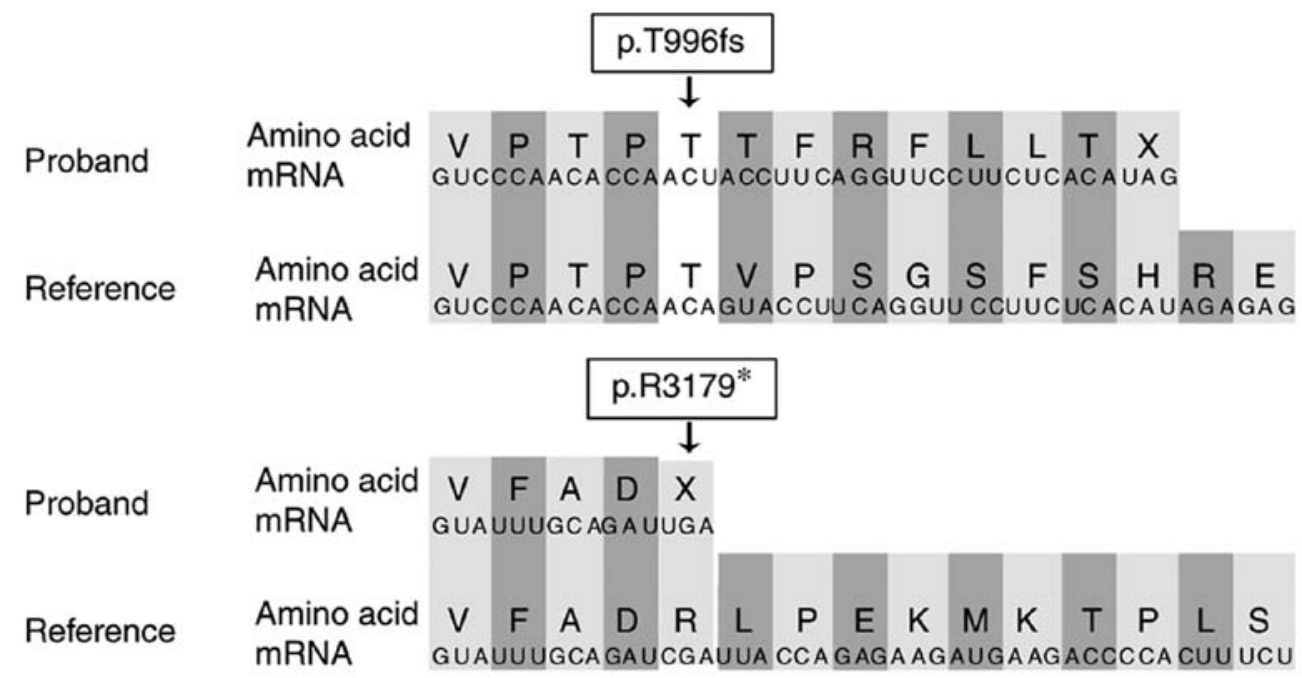

Figure 4. Amino acid and mRNA sequences of Alström syndrome protein 1 and the predicted premature termination codons.

MasterMix (cat. no. KK4824; Kapa Biosystems; Roche Diagnostics) and primers targeting the Illumina P5 and P7 flow cell oligo sequences. Library Quantification Primer Premix (10X), contained the following primers: Primer 1, 5'-AATGAT ACGGCGACCACCGA-3' and Primer 2,5'-CAAGCAGAAGAC GGCATACGA-3'. The PCR protocol included initial denaturation at $95^{\circ} \mathrm{C}$ for $5 \mathrm{~min}$ and then 35 cycles of $95^{\circ} \mathrm{C}$ for $30 \mathrm{sec}$ followed by $60^{\circ} \mathrm{C}$ for $30 \mathrm{sec}$. The average $\mathrm{Cq}$ score for each DNA Standard was plotted against $\log _{10}$ (concentration in $\mathrm{pM}$ ) to generate a standard curve. The concentrations of diluted library samples were then calculated against the standard curve using the $2^{-\Delta \Delta \mathrm{Cq}}$ method (9). The sequencing data were analyzed by BWA (version 0.7.10; https://www.plob.org/tag/bwa), ANNOVAR (version 1; http://annovar.openbioinformatics.org/en/latest/) and GATK (version 4.0.8.1; https://www.broadinstitute.org/gatk/) software as well as the public databases $1000 \mathrm{G}$ (http://browser. 1000genomes.org), ExAC (http://exac.broadinstitute.org/), gnomAD (http://gnomad.broadinstitute.org/), Esp6500 (http://evs.gs.washington.edu/EVS) and human genome mutation database (HGMD; https://portal.biobase-international. com/cgi-bin/portal/login.cgi. Additionally, the American College of Medical Genetics and Genomics (ACMG) guidelines (10) 
Table I. Summary of targeted next-generation sequencing on the patient.

\begin{tabular}{ll}
\hline Statistics data indicators & Statistical results \\
\hline Raw databases, $\mathrm{Mb}$ & $1,463.69155$ \\
Clean databases, $\mathrm{Mb}$ & $1,411.56$ \\
Aligned bases, $\mathrm{Mb}$ & $1,392.32$ \\
Aligned, \% & 98.64 \\
Initial bases on target, $\mathrm{n}$ & 564,206 \\
Base covered on target, $\mathrm{n}$ & 564,150 \\
Coverage of target region, $\%$ & 99.99 \\
Effective bases on target, $\mathrm{n}$ & $703,575,596$ \\
Fraction of effective & 50.53 \\
bases on target, $\%$ & $1,247.02$ \\
Average sequencing & \\
depth on target, $\mathrm{n}$ & 99.66 \\
Fraction of target covered & \\
with at least 20X, \% &
\end{tabular}

Mb, megabase.

Table II. Disease mutation of Alström syndrome protein 1 gene.

\begin{tabular}{lrr}
\hline Mutation type & No. of mutation & Ratio, \% \\
\hline Missense & 18 & 5.88 \\
Nonsense & 122 & 39.87 \\
Splicing substitutions & 7 & 2.29 \\
Small deletions/insertions/ & 148 & 48.37 \\
duplications & & \\
Gross deletions/insertions/ & 10 & 3.27 \\
duplications & & \\
Complex rearrangements & 1 & 0.33 \\
Total & 306 & 100.0 \\
\hline
\end{tabular}

were used for the interpretation of sequence variants. Furthermore, Sanger sequencing was used to verify the novel compound heterozygous mutations with two primer pairs to amplify c.2988_2989 and c.9535 of the ALMSI gene (reference genome version: GRCh37/hg19, reference transcript: NM_015120.2). The following primers were used: c.2988_2989 forward, 5'-ACCCTGCCAGACTTTCTTTT-3' and reverse, 5'-GCATAACTATCTGGCCACTCC-3'; c.9535 forward, 5'-TCCAAGTCGTACAGCCTTCT-3' and reverse, 5'- TCAGAG AAATCACACGGCCT-3'. Finally, Chromas software (version 2.4.1; Biosoft) was used to analyze the Sanger sequencing data.

Amniocentesis and prenatal diagnosis. Amniocentesis was performed at $21 \pm 2$ weeks of gestation. A total of $5 \mathrm{ml}$ amniotic fluid sample was taken for prenatal diagnosis of ALMS1. Fetal exfoliated cells in the amniotic fluid were collected by centrifugation (400 x g; $10 \mathrm{~min}$, room temperature) and washed with phosphate buffer saline. gDNA was extracted with the DNeasy Blood and Tissue kit (Qiagen $\mathrm{GmbH}$ ) according to the manufacturer's protocol. Short tandem repeat analysis (11) of the amniotic fluid cells and parental peripheral blood was used to confirm the relationship between the samples and also to confirm that there was no maternal cell pollution in the amniotic fluid. Sanger sequencing (12) was used to analyze the nucleotide sequences of the ALMS1 gene c.9535 and c.2988_2989.

\section{Results}

The average sequencing depth was $1247.02 \mathrm{X}$ on the targeted regions, and 1,546 variants in the disease-targeted gene panel of the proband were identified. The summary of the disease-targeted next generation sequencing (NGS) were listed in Table I. A total of two pathological variants in ALMS1 were observed: c.2988_2989delAG (chr2-73676644 73676646) in exon 8 and c.9535C $>\mathrm{T}$ (chr2-73718624) in exon 10. These variants were further verified by Sanger sequencing and the results demonstrated that c.2988_2989delAG (p.T996fs) was paternally inherited and c.9535C >T (p.R3179*) was maternally inherited (Fig. 3). These mutations lead to premature termination codons (Fig. 4).

The present study also observed that there was a heterozygous variation at c. $9535 \mathrm{C}>\mathrm{T}$ of the $A L M S 1$ gene in amniotic fluid cells, and there was no mutation at c.2988_2989 (Fig. 3). In addition, the fetus was followed-up for $>2$ years. The mother of the proband delivered a $3.5 \mathrm{~kg}$ male baby at $40 \pm 3$ weeks of gestation. The baby was examined by the children's health care department on a monthly basis, and had no abnormal phenotypes until 15 months of age. The mother of the proband did not wish for blood samples to be taken from the baby after birth to confirm the prenatal test results.

\section{Discussion}

The most common symptoms of AS are cone-rod dystrophy, sensorineural hearing loss, short stature, obesity, cardiomyopathy and metabolic disorders (13-16). The clinical symptoms, which are age of onset and severity, vary greatly among patients (2)

Currently, clinical diagnostic standard for AS are based on the major and minor criteria. The criteria include a pathogenic mutation in one ALMS1 allele, and/or family history of AS and vision problems. The minor criteria include obesity and/or insulin resistance and/or type 2 diabetes mellitus, dilated cardiomyopathy with congestive heart failure and hearing loss (2). However, diagnosis of AS is difficult because some symptoms (such as nystagmus and photophobia) start at birth whereas others (such as hypertension and renal failure) occur as the child grows $(2,17)$. Genetic testing should be performed when the criteria fail to allow for a clear clinical diagnosis (17). Due to the size and mutation spectrum of $A L M S 1$, the mutation analysis of $A L M S 1$ by conventional Sanger sequencing is usually complicated $(2,6)$. This difficulty can be resolved by the application of NGS.

Disease-targeted gene panel is often used for specific suspected disease or a group of diseases (18). Disease-targeted gene panels often have higher diagnostic rates compared with 
those of exome sequencing or genome sequencing, being designed to maximize coverage, sensitivity and specificity for the included genes (19). The cost of disease-targeted gene panels is variable but is often lower compared with that of exome sequencing and genome sequencing (19). In the present study, the patient was diagnosed with a high clinical suspicion of AS, and two compound heterozygous mutations in ALMS1 were detected using a disease-targeted gene panel.

Mutations are highly heterogeneous within ALMS1 (17). According to the statistics of HGMD (https://portal. biobase-international.com/cgi-bin/portal/login.cgi), a total of 306 disease-causing variants of ALMS1 have been reported, 285 of which are related to the syndrome phenotype. The majority of the variants are nonsense mutations $(39.87 \%)$ and frameshift mutations (48.37\%; Table II), and most of the mutations occur in exon 8,10 and $16(6,20)$. In this case, an unreported mutation: c.2988_2989del (p.T996fs), which was a frameshift mutation located in exon 8 , was identified. The c.9535C > T (p.R3179*), a nonsense mutation located in exon 10, was previously reported as a disease mutation variant $(6,21)$ in the HGMD database. Flintoff and Boute-Benejean (21) reported the variant p.R3179* as a novel pathological mutation without any details. Marshall et al (6) evaluated a large cohort of patients (including 250 affected individuals) with AS for variants in the ALMS1 gene and reported 55 novel variants which included p.R3179*. However, Marshall et al (6) only stated that p.R3179* was a novel variant, but did not indicate whether the variant was a homozygous or compound heterozygous, and whether the variants were from the proband's parents. This is very important according to the diagnostic criteria for AS (2). In addition, the authors did not specifically describe the clinical phenotype of the patient carrying p.R3179*. In the present study, the specific phenotypes of the patient was described, the variant (p.R3179*) was verified by NGS (the sequencing depth was 373X) and Sanger sequencing was used to verify the authenticity of the variant and whether the parents were also carriers. Combined with the clinical phenotype and gene test results of the patient, the patient was diagnosed with AS. The diagnosis of AS was very important as the patient was not diagnosed by molecular and genetic techniques until the age of 10 years. The diagnosis was crucial at that time due to his mother's pregnancy and desire for a prenatal diagnosis, which was made possible. The present study demonstrated that p.R3179* is a pathogenic variant in patients with AS, and prenatal diagnosis was performed for this variant, to the best of our knowledge, for the first time. Sequence analysis performed in the current study demonstrated that the mutations resulted in code shifting and nonsense mutations, which lead to premature termination codons. According to the criteria of the ACMG (10), the mutations were pathogenic, and the evidence for this was as follows: i) Very strong evidence of pathogenicity: Nonsense, frameshift; ii) moderate evidence of pathogenicity: Absent from controls in 1000G; and iii) supporting evidence of pathogenicity: Patient's phenotype is highly specific for a disease with a single genetic etiology (10).

According to the recommended clinical diagnostic criteria for AS, patients aged 3-14 years should fulfill two major criteria or one major and three minor criteria (2). The patient in the current study was a 10 -year-old male and fulfilled two major criteria (compound heterozygous mutations in ALMS1 and vision problems including photophobia and visual impairment) in addition to some minor criteria (obesity and hearing loss). Therefore, the diagnosis of AS was determined in the proband. However, due to the rarity of AS, delay in onset of some of the cardinal features, inter and intra familial clinical heterogeneity, some minor criteria clinical findings were not observed in the proband, such as renal failure (2). Marshall et al (6), demonstrated that a significant association between alterations in exon 8 and absent, mild or delayed renal disease may be due to the tissue-specific expression of different splicing subtypes or the lack of another subtype of exon 8 in the kidney, which may protect patients with exon 8 mutations from serious renal complications. This was consistent with the phenotype of the proband; the frameshift variant in the current study was in exon 8 , and the renal function (creatinine and blood urea nitrogen) of the patient was normal, without any renal disease. The case is still in the follow-up of the genetic counseling clinic. However, although the renal function was normal, renal ultrasound was not performed. Thus, the renal function of the patient should be actively monitored in future.

Patients with AS rarely live $>40$ years (22). At present, there is no specific treatment for AS, but early diagnosis and intervention can alleviate the progress of the disease phenotypes and improve the survival period and quality of life of patients (2). Early diagnosis can be treated by multi-disciplinary combination therapy (2). For patients with retinopathy, heart disease, obesity, deafness and diabetes, the possibility of AS should be considered and gene detection should be performed as early as possible for a clear diagnosis, which is conducive to the early assessment of the disease in order to improve the prognosis and long-term survival. For pregnant women with a positive AS family history, genetic counseling and prenatal diagnosis should be performed.

In the present study, a Chinese patient presenting clinical features compatible with AS was described. Using a disease-targeted gene panel, two compound heterozygous variants in the exons 8 and 10 were identified. Both the mutations were predicted to lead to premature termination codons, which may result in truncated ALMS1 protein formation. In addition, the specific DNA sequences of the two mutation sites were tested for in the fetus of the proband's mother.

This study identified two compound heterozygous ALMS1 mutations in a patient with the symptoms of AS and reported a novel ALMS1 variant which expands the spectrum of ALMS1 variants in AS.

\section{Acknowledgements}

Not applicable.

\section{Funding}

The current study was supported by the National Key Research and Development Program of China (grant no. 2018YFC1002200) and the Program of Science and Technology Department of Sichuan Province (grant no. 2020YFS0095). 


\section{Availability of data and materials}

All data generated or analyzed during this study are included in this published article.

\section{Authors'contributions}

CZ, SL and JW designed the study. CZ, YX and HX performed the experiments. CZ and JW conducted data analysis. All authors read and approved the final manuscript.

\section{Ethics approval and consent to participate}

The present study was approved by the Medical Ethics Committee of West China Second University Hospital of Sichuan University. Written informed consent to participate was obtained from the parents of the patient.

\section{Patient consent for publication}

Written informed consent for publication was obtained from the parents of the patient.

\section{Competing interests}

The authors declare that they have no competing interests.

\section{References}

1. Alström CH, Hallgren B, Nilsson LB and Asander H: Retinal degeneration combined with obesity, diabetes mellitus and neurogenous deafness: a specific syndrome (not hitherto described) distinct from the Laurence-Moon-Bardet-Biedl syndrome: a clinical, endocrinological and genetic examination based on a large pedigree. Acta Psychiatr Neurol Scand Suppl 129: 1-35, 1959.

2. Marshall JD, Beck S, Maffei P and Naggert JK: Alström syndrome. Eur J Hum Genet 15: 1193-1202, 2007.

3. Marshall JD, Bronson RT, Collin GB, Nordstrom AD, Maffei P, Paisey RB, Carey C, Macdermott S, Russell-Eggitt I, Shea SE, et al: New Alström syndrome phenotypes based on the evaluation of 182 cases. Arch Intern Med 165: 675-683, 2005.

4. Hearn T, Renforth GL, Spalluto C, Hanley NA, Piper K, Brickwood S, White C, Connolly V, Taylor JF, Russell-Eggitt I, et al: Mutation of ALMS1, a large gene with a tandem repeat encoding 47 amino acids, causes Alström syndrome. Nat Genet 31: 79-83, 2002.

5. Collin GB, Marshall JD, Cardon LR and Nishina PM: Homozygosity mapping at Alström syndrome to chromosome $2 \mathrm{p}$. Hum Mol Genet 6: 213-219, 1997.

6. Marshall JD, Hinman EG, Collin GB, Beck S, Cerqueira R, Maffei P, Milan G, Zhang W, Wilson DI, Hearn T, et al: Spectrum of ALMS1 variants and evaluation of genotype-phenotype correlations in Alström syndrome. Hum Mutat 28: 1114-1123, 2007.

7. Collin GB, Marshall JD, King BL, Milan G, Maffei P, Jagger DJ and Naggert JK: The Alström syndrome protein, ALMS1, interacts with $\alpha$-actinin and components of the endosome recycling pathway. PLoS One 7: e37925, 2012.
8. Hearn T: ALMS1 and Alström syndrome: A recessive form of metabolic, neurosensory and cardiac deficits. J Mol Med (Berl) 97: 1-17, 2019

9. Livak KJ and Schmittgen TD: Analysis of relative gene expression data using real-time quantitative PCR and the $2(-\Delta \Delta$ C(T)) Method. Methods 25: 402-408, 2001.

10. Richards S, Aziz N, Bale S, Bick D, Das S, Gastier-Foster J, Grody WW, Hegde M, Lyon E, Spector E, et al; ACMG laboratory quality assurance committee: Standards and guidelines for the interpretation of sequence variants: A joint consensus recommendation of the American College of Medical Genetics and Genomics and the Association for Molecular Pathology. Genet Med 17: 405-424, 2015.

11. Wang J, Chen L, Zhou C, Wang L, Xie H, Xiao Y, Zhu H, Hu T, Zhang Z, Zhu Q, et al: Prospective chromosome analysis of 3429 amniocentesis samples in China using copy number variation sequencing. Am J Obstet Gynecol 219: 287.e1-287.e18, 2018.

12. Beck TF and Mullikin JC; NISC Comparative Sequencing Program, Biesecker LG: Systematic evaluation of Sanger validation of next-generation sequencing variants. Clin Chem 62: 647-654, 2016.

13. Casey J, McGettigan P, Brosnahan D, Curtis E, Treacy E, Ennis S and Lynch SA: Atypical Alstrom syndrome with novel ALMS1 mutations precluded by current diagnostic criteria. Eur J Med Genet 57: 55-59, 2014.

14. Russell-Eggitt IM, Clayton PT, Coffey R, Kriss A, Taylor DS and Taylor JF: Alström syndrome. Report of 22 cases and literature review. Ophthalmology 105: 1274-1280, 1998.

15. Waldman M, Han JC, Reyes-Capo DP, Bryant J, Carson KA, Turkbey B, Choyke P, Naggert JK, Gahl WA, Marshall JD, et al: Alström syndrome: Renal findings in correlation with obesity, insulin resistance, dyslipidemia and cardiomyopathy in 38 patients prospectively evaluated at the NIH clinical center. Mol Genet Metab 125: 181-191, 2018.

16. Collin GB, Marshall JD, Ikeda A, So WV, Russell-Eggitt I, Maffei P, Beck S, Boerkoel CF, Sicolo N, Martin M, et al: Mutations in ALMS1 cause obesity, type 2 diabetes and neurosensory degeneration in Alström syndrome. Nat Genet 31: 74-78, 2002.

17. Marshall JD, Maffei P, Collin GB and Naggert JK: Alström syndrome: Genetics and clinical overview. Curr Genomics 12: 225-235, 2011.

18. Pulignani S, Vecoli C, Borghini A, Foffa I, Ait-Alì L and Andreassi MG: Targeted next-generation sequencing in patients with non-syndromic congenital heart disease. Pediatr Cardiol 39: 682-689, 2018.

19. Adams DR and Eng CM: Next-generation sequencing to diagnose suspected genetic disorders. N Engl J Med 379: 1353-1362, 2018.

20. Marshall JD, Muller J, Collin GB, Milan G, Kingsmore SF, Dinwiddie D, Farrow EG, Miller NA, Favaretto F, Maffei P, et al: Alström syndrome: Mutation spectrum of ALMS1. Hum Mutat 36: 660-668, 2015.

21. Flintoff KJ and Boute-Benejean O: Novel human pathological mutations. Gene symbol: ALMS1. Disease: Alstrom syndrome. Hum Genet 121: 645-652, 2007.

22. Nerakh G and Ranganath P: Alström Syndrome presenting as isolated dilated cardiomyopathy. Indian J Pediatr 86: 296-298, 2019.

This work is licensed under a Creative Commons Attribution-NonCommercial-NoDerivatives 4.0 International (CC BY-NC-ND 4.0) License. 